Methods Patients diagnosed with vulvar SCC or VC between the years of 2004 and 2016 were identified in the NCDB. OS was assessed with Kaplan-Meier curves and the log-rank test. Construction of a Cox model compared survival after controlling for confounding variables.

Results The reported incidence of SCC of the vulva has significantly increased since $2004(\mathrm{p}<0.0001)$. In contrast, the incidence of VC has remained stable since $2004(\mathrm{p}=0.344)$. Compared to SCC, VC was significantly more likely to be diagnosed in older women $(p<0.0001)$ and treated with surgery alone $(\mathrm{p}<0.0001)$. However, on propensity score weighted analysis there was a trend toward improved OS in women with VC compared to those with SCC ( $p=0.0794)$. Multivariable Cox survival analysis showed an improvement in $\mathrm{OS}$ in VC patients treated with both primary site and regional lymph node surgery compared to primary site surgery alone (HR 0.67, 95\% confidence interval [CI] $0.46-0.97, \mathrm{p}=$ 0.0357).

Conclusions Verrucous carcinoma is more likely to present in women at an older age. Regional lymph node surgery in addition to primary site surgery significantly improves $\mathrm{OS}$ in $\mathrm{VC}$ patients.

\section{EPV289/\#657 EPIDEMIOLOGICAL PROFILE OF PATIENTS WITH MALIGNA VULVA NEOPLASIA ATTENDED AT SANTA MARCELINA ITAQUERA HOSPITAL - SAO PAULO}

${ }^{1} \mathrm{JA}$ Barbosa*, ${ }^{2} \mathrm{M}$ Mesquita, ${ }^{2} \mathrm{~S}$ Sanches, ${ }^{2} \mathrm{C}$ Sousa, ${ }^{2} \mathrm{M}$ Silva, ${ }^{2} \mathrm{M}$ Manchini, ${ }^{2} \mathrm{M}$ Brandão, ${ }^{2} \mathrm{C}$ Gomez, ${ }^{2} \mathrm{~T}$ Almeida. ${ }^{1} \mathrm{Casa}$ de Saúde Santa Marcelina, Gynecology Oncology, Sao Paulo, Brazil; ${ }^{2}$ Casa de Saúde Santa Marcelina, Gynecologic Oncology, Sao Paulo, Brazil

\subsection{6/ijgc-2021-IGCS.360}

Objectives Objective: To study the epidemiological profile of patients diagnosed with vulvar malignancy seen at Santa Marcelina Itaquera Hospital (HSM) in São Paulo.

Methods Analysis of medical records of patients undergoing follow-up at the Gynecology Oncology outpatient clinic between the years 2008 to 2020. The information analyzed were: age, parity, smoking, histological type of the tumor, neoadjuvance, surgical treatment, adjuvance, recurrence, lymph node involvement and death.

Results

Result 45 patients were seen, whose average age was 66 years, which numerically represents $51.11 \%$ of the patients seen; $26.66 \%$ were smokers and the most common histological type is squamous cell carcinoma, marking $82.22 \%$ of all other types identified. Five patients $(11.11 \%)$ were classified as stage I, fifteen (33.33\%) stage II, thirteen (28.88\%) stage III and twelve (26.66) of stage IV patients. Within this scenario, neoadjuvant therapy was part of $60 \%$ of the cases; surgical treatment $80 \%$ and adjuvance $62.22 \%$. Sixty-four percent of the patients did not experience recurrence or disease progression. Forty percent of patients who did surgical procedure with lymphadenectomy had lymph node involvement and twentyfour patients $(53.33 \%)$ died.

Conclusions

Conclusion The epidemiological profile of patients are consistent with the literature, from the age group, histological type, percentage of death and recurrence. The high rate of death is mainly related to late diagnosis, although neoadjuvant treatment allows surgery in advanced cases.

\section{EPV290/\#90 LONG-TERM RESULTS OF PRIMARY VAGINAL CANCER TREATMENT: THE BELARUS NATIONAL CANCER CENTRE EXPERIENCE}

${ }^{1} \mathrm{O}$ Matylevich*, ${ }^{2} \mathrm{H}$ Trukhan, ${ }^{1} \mathrm{E}$ Dolomanova, ${ }^{1} \mathrm{D}$ Rouski, ${ }^{3} \mathrm{O}$ Zubets, ${ }^{1} \mathrm{~S}$ Mavrichev. ${ }^{1} \mathrm{NN}$ Alexandrov National Cancer Centre of Belarus, Gynecologic Oncology Department, Minsk, Belarus; ${ }^{2}$ Belarusian Medical Academy of Postgraduate Education, Department of Oncology, Minsk, Belarus; ${ }^{3}$ NN Alexandrov National Cancer Centre of Belarus, Cancer Control Department, Minsk, Belarus

\subsection{6/ijgc-2021-IGCS.361}

Objectives To study the long-term results of treatment of vaginal cancer (VC) patients and to evaluate the results of diagnosis and treatment of patients living in urban and rural areas.

Methods The data of 70 patients with primary VC treated at NN Alexandrov National Cancer Centre of Belarus from 2000 to 2019 were included. The median age was 64 years (rang, 32-87). Morphology in 91.5\% (64/70) cases was squamous cell cancer, in $7.1 \%(5 / 70)$ - adenocarcinoma, in $1.4 \%$ $(1 / 70)$ - adenosquamous carcinoma. The distribution by the stage was as follows: Stage I in $17(24.3 \%)$ patients, Stage II in $30(42.9 \%)$, Stage III in $12(17.1 \%)$, Stage IV in 11 $(15.7 \%)$ cases. Treatment was performed in $82.8 \%(58 / 70)$ cases: in $94.1 \%(16 / 17)$ for Stage I disease, in $83.3 \%(25 / 30)$ for Stage II, in $91.7 \%(11 / 12)$ for Stage III, and in $54.5 \%$ (6/ 11) for Stage IV.

Results The median follow-up time was 33 months (range, 1220). A total of 42 women died: 28 from progression of VC and 14 from other diseases. Overall survival (OS) was 31.9 $\pm 6.8 \%$, median survival - 41 months (95\% CI 0.0-105.3). Disease-specific survival (DSS) for the entire group was 54.5 $\pm 6.8 \%$, median not reached. The overall survival rate of urban women was $44.8 \pm 10.6 \%$, rural $-22.5 \pm 8.2 \%$, p = 0.142 ; DSS $-57.6 \pm 10.5 \%$ and $53.0 \pm 8.4 \%, \mathrm{p}=0.448$, respectively.

Conclusions The DSS rate was $54.0 \pm 6.8 \%$; the OS rate did not exceed $31.9 \pm 6.8 \%$. Rural residence was not associated with late stage at diagnosis or receipt of treatment.

\section{EPV293/\#425 EFFECT ON OVERALL SURVIVAL OF CANCER PROGRAM-LEVEL VARIATION IN THE USE OF NEOADJUVANT CHEMOTHERAPY FOR ADVANCED OVARIAN CANCER: A DIFFERENCE-IN- DIFFERENCES STUDY}

\begin{abstract}
${ }^{1}$ A Melamed*, ${ }^{2} \mathrm{JA}$ Rauh-Hain, ${ }^{1} \mathrm{~A}$ Gockley, ${ }^{2} \mathrm{R}$ Nitecki, ${ }^{3} \mathrm{P}$ Ramirez, ${ }^{4} \mathrm{D}$ Hershman, ${ }^{5} \mathrm{~N}$ Keating, ${ }^{1} \mathrm{~J}$ Wright. ${ }^{1}$ Columbia University College of Physicians and Surgeons, Gynecologic Oncology, New York City, USA; ${ }^{2}$ University of Texas MD anderson Cancer Center, Gynecologic Oncology, Houston, USA; ${ }^{3}$ University of Texas MD anderson Cancer Center, Gynecologic Oncology and Reproductive Medicine, Houston, USA; ${ }^{4}$ Herbert Irving Comprehensive Cancer Center, Oncology, New York City, USA; ${ }^{5}$ Harvard Medical School, Health Policy, Boston, USA
\end{abstract}

\subsection{6/ijgc-2021-IGCS.362}

Objectives To evaluate the effect of cancer program-level variations in use of neoadjuvant chemotherapy (NACT) on overall survival among patients with advanced ovarian cancer.

Methods We included women with advanced-stage epithelial ovarian cancer treated 2004-2015 in Commission on Canceraccredited cancer programs that began administering NACT liberally or continued to restrict its use after the publication of a randomized trial in 2010. We used flexible parametric survival models to perform a difference-in-differences analysis 
evaluating the effect of liberal NACT administration on casemix-standardized median overall survival and 1-year mortality rates.

Results We identified 19,562 patients treated in 332 cancer programs that increased use of NACT from $21.7 \%$ in 20042009 to $42.2 \%$ in $2010-2015$ and 19,737 patients treated in 332 programs that marginally increased use of NACT $(20.1 \%$ to $22.5 \%$ ) over the same period. Standardized median overall survival improved by similar magnitudes in programs with liberal (from 31.6 to 37.9 months; 6.3-month difference; 95\% CI, 4.2-8.3) and restrictive (from 31.4 to 36.8 months; 5.4month difference, 95\% CI, 3.5-7.3) use of NACT after 2010 (difference-in-differences, 0.9 months; $95 \% \mathrm{CI},-1.9$ to 3.7 ). One-year mortality declined more in programs with liberal (from $25.6 \%$ to $19.3 \%$; risk difference, $-5.2 \%$; $95 \%$ CI, -6.4 to -4.1 ) than with restrictive (from $24.9 \%$ to $21.8 \%$; risk difference, $-3.2 \%, 95 \% \mathrm{CI},-4.3$ to -2.0$)$ use of NACT (difference-in-differences, $-2.1 \% ; 95 \% \mathrm{CI},-3.7$ to -0.5 ).

Conclusions Compared with cancer programs that administered NACT restrictively, those that administered it liberally achieved similar improvements in median overall survival and larger declines in short-term mortality.

\section{EPV295/\#178 EFFICACY AND TOLERABILITY OF WEEKLY PACLITAXEL AS 'SALVAGE THERAPY' IN PATIENTS WITH GYNECOLOGICAL TUMORS}

${ }^{1} \mathrm{M}$ Ligorio, ${ }^{1} \mathrm{~F}$ Arezzo, ${ }^{2} \mathrm{~V}$ Loizzi, ${ }^{1} \mathrm{E}$ Cicinelli, ${ }^{1} \mathrm{M}$ Spinelli, ${ }^{1} \mathrm{G}$ Sicolo, ${ }^{1} \mathrm{G}$ Cormio*, ${ }^{1} \mathrm{R}$ De Nola. ${ }^{1}$ University Hospital Polyclinic of Bari: Azienda Ospedaliero-Universitaria Consorziale Policlinico di Bari, Obstetrics and Gynecology Department, Policlinico of Bari, Bari, Italy; ${ }^{2}$ University of Bari 'Aldo Moro', Interdisciplinar Department of Medicine, Bari, Italy

\subsection{6/ijgc-2021-IGCS.363}

Objectives Despite paclitaxel has been used routinely in gynecological tumors, there are still few studies in literature that have investigated its efficacy and tolerability as salvage therapy. The aim of this study is to examine weekly paclitaxel's efficacy and tolerability as salvage therapy in patients diagnosed with gynecological tumors.

Methods A retrospective study was conducted on 96 patients diagnosed in our 'II Clinica Ginecologica' of Policlinico di Bari, between October 1992 and July 2019. Inclusion criteria were: 1) diagnosis of ovarian, endometrial, or cervical tumor 2) patients who received treatment with weekly paclitaxel as salvage therapy To evaluate the efficacy, PFS and OS were elaborated with Kaplan Meier curves and compared with Log Rank test. Response to therapy was also considered (stable disease or partial response vs progression of disease). Tolerability was evaluated collecting data about adverse events from medical records.

Results
Ovarian tumor 81/96 cases; OS median 13 months $(7,6-18,4)$; PFS median 17 weeks (12,8-21,1); positive response $68,6 \%$ Endometrial tumor: 9/96 cases; OS median 9 months (017,9); PFS median 18 weeks $(5,2-30,7)$; positive response $77,8 \%$ Cervical tumor: 6/96 cases; OS median 19 months $(10,4-27,5)$; PFS median 23 weeks $(0-50,9)$; positive response 66,7\% Toxicity: $18,4 \%$ anemia; $12,6 \%$ leucopenia; $3,4 \%$ peripheral neuropathy; $2,3 \%$ myalgia/arthralgia; $1,1 \%$ cardiac toxicity; $1,1 \%$ ocular toxicity; $2,3 \%$ thrombocytopenia.

Conclusions With this study, the efficacy of weekly paclitaxel as salvage therapy can be confirmed. Moreover, this treatment is well tolerated by patients.

\section{EPV296/\#395 'A PROSPECTIVE MULTICENTRIC STUDY OF RISK- REDUCING SALPINGO-OOPHORECTOMY IN BRCA MUTATION PATIENTS'}

${ }^{1} \mathrm{M}$ Spinelli, ${ }^{2} \mathrm{~F}$ Arezzo, ${ }^{3} \mathrm{G}$ Sicolo, ${ }^{3} \mathrm{CM}$ Santarsiero, ${ }^{4} \mathrm{~V}$ Loizzi, ${ }^{5} \mathrm{G}$ Cazzato, ${ }^{5} \mathrm{~L}$ Resta, ${ }^{5} \mathrm{G}$ Serio, ${ }^{6} \mathrm{G}$ Cormio* ${ }^{7}{ }^{7}$ E Cicinelli, ${ }^{2}$ R Romagno. ${ }^{1}$ Policlinico of Bari, Obstetrics and Gynecology Department, Bari, Italy; ${ }^{2}$ Policlinico of Bary, Obstetrics and Gynecology Department, Bari, Italy; ${ }^{3}$ university of Bari Aldo Moro', Biomedical Sciences and Human Oncology Obtetrics and Gynecology Unit, BARI, Italy; 'University of Bari 'Aldo Moro', Interdisciplinar Department of Medicine, Bari, Italy; ${ }^{5}$ university of Bari Aldo Moro', Emergency and Organ Transplantation, Pathology Section, BARI, Italy; ${ }^{6}$ University of Bari 'Aldo Moro', Department of Biomedical Sciences and Human Oncology, Bari, Italy; 'University of Bari 'Aldo Moro', Department of Biomedical Science and Human Oncology, Gynecology and Obstetrics Section, Bari, Italy

\subsection{6/ijgc-2021-IGCS.364}

Objectives BRCA1/2 are tumour-suppressor genes involved in DNA homologous recombination and ovarian cancer development.

Methods Risk-reducing surgery (RRS) was performed in 148 patients carrying BRCA1 (aged between 30-73 years, median age was 51 years) and BRCA 2 mutation (aged between 3670 years, median age was 53 years). Seventy-nine patients had previous history of breast cancer.

Results Between the all patients, 131 women underwent riskreducing salpingo-oophorectomy (RRSO) through a laparoscopic minimally invasive approach, $11(7,4 \%)$ underwent laparoscopic RRSO and contextual hysterectomy, 2 woman $(1,3 \%)$ underwent RRSO through a laparotomic approach and 12 women $(8,1 \%)$ laparotomic RRSO and hysterectomy. During 7 (4,7\%) laparoscopic RRSO, prophylactic bilateral mastectomy was also performed. Early and late complication occurred in 4 patients (3\%). Six patients (4\%) were found to have occult Serous Tubal Intraepithelial Carcinoma (STIC) and seven patients $(4,7 \%)$ occult cancer.

Conclusions RRSO is safe and feasible in BRCA mutation carriers. The procedure is effective for genetic prevention of ovarian cancer. 